\title{
SUCCESSFUL OUTCOME OF PREGNANCY WITH APLASTIC ANAEMIA: A CASE REPORT
}

Sunil Kumar K. S, Laxmi V. Yaliwal, R. M. Desai, Prajna Kumari, Apurva. S. A.

1. Associate Professor. Department of Obstetrics \& Gynaecology, SDM College of Medical Sciences \& Hospital,

2. Associate Professor. Department of Obstetrics \& Gynaecology, SDM College of Medical Sciences \& Hospital,

3. Professor. Department of Obstetrics \& Gynaecology, SDM College of Medical Sciences \& Hospital,

4. Post Graduate. Department of Obstetrics \& Gynaecology, SDM College of Medical Sciences \& Hospital,

5. Post Graduate. Department of Obstetrics \& Gynaecology, SDM College of Medical Sciences \& Hospital,

\section{CORRESPONDING AUTHOR:}

Dr. Sunil Kumar K. S,

Associate Professor,

Dept. of OBG, SDMCMSH,

Sattur, Dharwad, PIN 580009.

E-mail: drsuneelks@yahoo.com

SUMMARY: Pregnancy in aplastic anaemia is rare and it offers great problems to haematologist as well as obstetrician as the management of such cases challenges their skills in deciding the best treatment option for the patient. It could be life threatening because of the haemorrhage and sepsis in the mother while the foetus may suffer from intrauterine growth restriction and intrauterine death. However the gold standard treatment for aplastic anaemia is bone marrow transplantation, which is contraindicated in pregnancy because it requires high doses of immunosuppressive therapy which could be toxic to the foetus. There is no universal agreement about the optimal treatment regime for this disorder in pregnancy. The present case report describes a patient with aplastic anaemia who was managed with intensive haematological support leading to delivery of a healthy infant by caesarean section.

KEY WORDS: aplastic anaemia, immunosuppressive therapy, pregnancy outcome

INTRODUCTION: Aplastic anaemia is a syndrome of bone marrow failure defined by pancytopenia and bone marrow hypocellularity. Patients with aplastic anaemia have pallor and fatigue due to anaemia, bruising and bleeding due to thrombocytopenia and recurrent infections as a result of neutropenia. Causative agents include T-cell mediated auto-immune disease, iatrogenic agents, viral infection and pregnancy. The relationship between aplastic anaemia and pregnancy is uncertain, but the literature suggests that there is no strong causal association in most cases ${ }^{1}$. However, Pregnancy in aplastic anaemia is very rare; it may exacerbate bone marrow depression and cause deterioration. Since only a handful of cases been reported so far, there is no clear guidelines established for the management of aplastic anaemia during pregnancy. Mortality in aplastic anaemia associated with pregnancy is $20-60 \%{ }^{2}$. The outcome in pregnancy is generally good owing to the recent advances in the supportive therapy, and up to $39 \%$ will have successful pregnancies².

CASE REPORT: Our patient is 26yrs old primigravida visited to us first time at 33wks 1 day, came with history of bleeding per vaginum about $100 \mathrm{ml}$ without any cause. She was a known case of aplastic anaemia diagnosed 7years back on bone marrow examination. Her haemoglobin at that time was $3 \mathrm{gm} \%$, and total counts were 3984cells/dl, and her platelets were 28,000 . She had received multiple blood transfusions and she was put on prednisolone $20 \mathrm{mg}$ per day. 
Patient stopped prednisolone on her own to receive ayurvedic preparations for the last 5 years and no blood tests were done prior to conception. On admission her vitals were stable except for presence of pallor, blood pressure was normal. No active bleeding was seen per vaginum. Ultrasound showed no evidence of placenta previa or abruption. Her haemoglobin was $8 \mathrm{~g} / \mathrm{dl}$, WBCs 4030cells/dl and platelets were 49000cells/dl. Coagulation profile was normal.

She was put on prednisolone $40 \mathrm{mg}$ daily. Patient was given 4 units of platelet transfusions. Betamethasone was given for foetal lung maturity. Subsequently the patient developed severe preeclampsia requiring termination of pregnancy. Caesarean was done at 35 weeks in view of severe preeclampsia, indeterminate antepartum haemorrhage and unfavourable cervix. A live female baby weighing $1.7 \mathrm{~kg}$ was born with good APGAR score. Intraoperative, there was moderate bleeding and she received 2 units of platelet and one unit of whole blood transfusion. Post operative period was uneventful. Prednisolone was restarted after the delivery. Her platelets were 39000 cells/dl and haemoglobin was $8.1 \mathrm{gm}$ at time of discharge.

DISCUSSION: The concept of aplastic anaemia as a clinical entity was first described by Paul Ehrlich in 1888 in a pregnant woman who died within a month of diagnosis. Although rarely encountered during pregnancy, aplastic anemia is a grave complication. It is characterized by pancytopenia and markedly hypocellular bone marrow. More than $50 \%$ of the cases are idiopathic; aetiology can be identified in only a third of cases. Causes include drugs and other chemicals, infections, irradiation, leukaemia, immunological disorders, and inherited conditions such as Fanconi anaemia and Diamond-Blackfan syndrome ${ }^{3,4}$. The functional defect appears to be a marked decrease in committed stem cells.

After going through the literatures it appears that the relation between the pregnancy and aplastic anemia is still unclear. In most cases, aplastic anemia and pregnancy simultaneously occur by chance. In few cases aplastic anemia has been identified first during pregnancy and then improved or even resolved when pregnancy terminated, only to recur with subsequent pregnancies. If the disease pre-exists then pregnancy may contribute to worsening of aplastic anaemia, as seen in our case. It has been observed that pregnancy results in an increase in production of placental lactogen, erythropoietin and oestrogen. Placental lactogen and erythropoietin stimulate haematopoiesis and estrogens suppress the marrow. Based on these observations Fleming suggested that an imbalance between these hormones results in hypoplasia of marrow in pregnancy leading to worsening of the symptoms 5 .

The major risks to the pregnant woman with aplastic anaemia are haemorrhage and infection, while the foetus may suffer from growth restriction and even intrauterine death. Other antenatal complications that occur due to placental vascular etiologies are abortion, preeclampsia, still birth and preterm birth ${ }^{6}$. Our patient developed severe preeclampsia requiring termination of pregnancy. Fortunately we did not encounter haemorrhage either during caesarean or in the postpartum period.

There is no universal agreement over the optimal management of aplastic anaemia in pregnancy. Only a handful of research has been published about the therapy during pregnancy. The most effective treatment for aplastic anaemia in a non-pregnant woman is bone marrow transplantation with a 5 -year survival of 56 to $89 \%{ }^{7}$. However the bone marrow transplantation is generally not used during pregnancy because of the teratogenic effects of immunosuppressive agents on the growing foetus. Earlier case reports in 1989 by Aitcheson et al proposed termination of pregnancy in the first trimester for patients with aplastic anemia ${ }^{8}$. But now, 
supportive measures are improving with the availability of better transfusion services and hence pregnancies should be maintained as long as the health of the mother is not seriously affected. Perhaps the mainstay of the management of aplastic anaemia in pregnancy is supportive therapy with erythrocyte, platelets, and granulocyte transfusions ${ }^{9}$. If the patient did not respond sufficiently then antithymocyte immunoglobulin, cyclosporine and steroids should be started 10,11 . There are several case reports of successful pregnancies in women who had undergone bone marrow transplantation ${ }^{12}$. There is a potential for umbilical cord-derived stem cell transplantation in the future ${ }^{13}$.

CONCLUSION: Aplastic anaemia is a serious medical disorder when associated with pregnancy. At present, we cannot draw any conclusions regarding the optimal therapies other than supportive therapy for patients with aplastic anaemia in pregnancy depending on few case reports. It is possible to have a successful outcome in moderately severe aplastic anaemia and severe thrombocytopenia through pregnancy with recent advances in supportive care and blood transfusion. Antithymocyte globulin, cyclosporine, and steroids are to be used in refractory cases although definite evidence for their use and efficacy in pregnancy is lacking.

\section{REFERENCES:}

1. Oosterkamp HM, Brand A, Kluin-Nelemans JC, Vandenbroucke JP: Pregnancy and severe aplastic anaemia: causal relation or coincidence? Br J Haematol 1998, 103:315-316.

2. Kuispel JW, Lynch VA, Vicle ED. Aplastic anemia in pregnancy a case report, review of literature and a re-evaluation of management. Obstet. Gynecol., Surv., 1976:31:523-28.

3. Green AM, Kupfer GM: Fanconi anemia. Hematol Oncol Clin North Am 23(2):193, 2009

4. Lipton JM, Ellis SR: Diamond-Blackfan anemia: Diagnosis, treatment, and molecular pathogenesis. Hematol Oncol Clin North Am 23(2):261, 2009

5. Choudhry VP, Gupta S, Gupta M, et al: Pregnancy associated aplastic anemia-A series of 10 cases with review of literature. Hematol 7:233, 2002

6. Faivre L, Meerpohl J, Da Costa L, et al: High-risk pregnancies in Diamond-Blackfan anemia: A survey of 64 pregnancies from the French and German registries. Haematologica 91:530, 2006

7. Young NS: Acquired aplastic anemia. Ann Intern Med 2002, 136:534-546

8. Aitchison RG, Marsh JC, Hows JM, Russell NH, Gordon-Smith EC: Pregnancy associated aplastic anaemia: a report of five cases and review of current management. $\mathrm{Br} \mathrm{J}$ Haematol 1989, 73:541-545.

9. Kwon JY, Lee Y, Shin JC, Lee JW,RhaJG,Kim SP.Supportive management of pregnancyassociated aplastic anemia. Int J Gynaecolobstet 2006;95(2):115-20

10. Krista JM Stibbe, Hajo IJ Wildschut, Pieternella J Lugtenburg. Management of Aplastic anemia in a woman during pregnancy: a case report. Journal of medical case reports 2011, 5:66.

11. Yilmaz E, et al. Aplastic Anemia and Pregnancy: Case Report: Perinatal Journal Vol: 15, Issue: 1/April 2007

12. Eliyahu S, Shalev E: A successful pregnancy after bone marrow transplantation for severe aplastic anaemia with pretransplant conditioning of total lymph-node irradiation and cyclophosphamide. Br J Haematol 86:649, 1994

13. Moise KJ Jr: Umbilical cord stem cells. Obstet Gynecol 106:1393, 2005 